EUROPHYSICS LETTERS

Europhys. Lett., (), pp. ()

\title{
Extension of Haff's cooling law in granular flows.
}

\author{
R. BRITO ${ }^{1}$ AND M.H. ERnst ${ }^{2}$ \\ ${ }^{1}$ Departamento de Física Aplicada I, Universidad Complutense de Madrid \\ 28040-Madrid, Spain \\ ${ }^{2}$ Instituut voor Theoretische Fysica, Universiteit Utrecht \\ Postbus 80006, 3508 TA Utrecht, The Netherlands
}

(received ; accepted )

PACS. 05.20.y - Statistical mechanics.

PACS. 05.20.Dd- Kinetic theory.

PACS. 81.05.Rm- Porous materials; granular materials.

\begin{abstract}
The total energy $E(t)$ in a fluid of inelastic particles is dissipated through inelastic collisions. When such systems are prepared in a homogeneous initial state and evolve undriven, $E(t)$ decays initially as $t^{-2} \sim \exp [-2 \epsilon \tau]$ (known as Haff's law), where $\tau$ is the average number of collisions suffered by a particle within time $t$, and $\epsilon=1-\alpha^{2}$ measures the degree of inelasticity, with $\alpha$ the coefficient of normal restitution. This decay law is extended for large times to $E(t) \sim$ $\tau^{-d / 2}$ in $d$-dimensions, far into the nonlinear clustering regime. The theoretical predictions are quantitatively confirmed by computer simulations, and holds for small to moderate inelasticities with $0.6<\alpha<1$.
\end{abstract}

A fluid of inelastic hard spheres (IHS) in $d$ dimensions, prepared initially in a state of thermal equilibrium with temperature $T_{0}$, will remain for an extended period of time (measured in average number of collisions suffered per particle) in a spatially homogeneous cooling state (HCS), provided the degree of inelasticity $\epsilon$ is small. In this state the average kinetic energy per particle $E(t)=\frac{d}{2} T(t)$ is decreasing like $1 / t^{2}$ due to inelastic collisions, as first explained by Haff [i]. However, the HCS with a spatially uniform density and temperature, and a vanishing flow field is unstable against long wavelength spatial fluctuations, and leads finally to a state with large scale clusters in the density field $n(\mathbf{r}, t)$ as well as in the flow field $\mathbf{u}(\mathbf{r}, t)$ (vortices). This is illustrated in the MD simulations of fig. 1. In this state cooling slows down and large deviations from Haff's law occur, which have not yet been explained in any quantitative manner [2, 3].

In general analytic results for nonlinear decay of rapid granular flows are rare [2]. The goal of this letter is to calculate the long time decay of $E(t)$ from nonlinear hydrodynamics, using mode coupling methods. The basic idea is to decompose the quantities of interest, here the total microscopic energy, or - in the study of long time tails of Green-Kubo formulas - the total microscopic momentum-, energy- or particle fluxes, into a superposition of products of slow hydrodynamic fluctuations of density $n(\mathbf{r}, t)$, temperature $T(\mathbf{r}, t)$ and flow field $\mathbf{u}(\mathbf{r}, t)$.

Typeset using EURO-TEX 

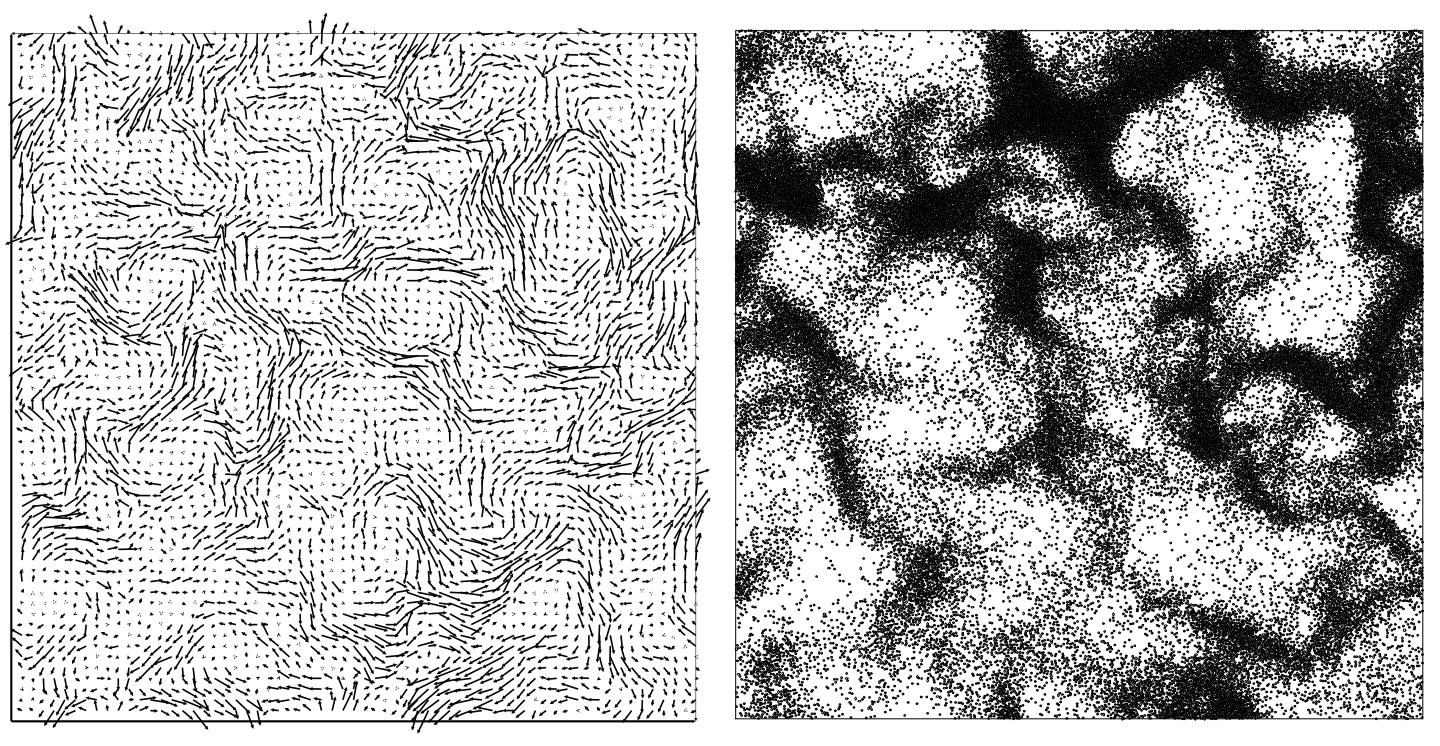

Fig. 1. -

Snapshots (a) of flow field $\mathbf{u}(\mathbf{r}, \tau=80)$ and (b) of density field $n(\mathbf{r}, \tau=160)$ in an IHS simulation with $N=50000, \alpha=0.9, \phi=0.245$, prepared in an initial thermal equilibrium state. Crossover to the nonlinear regime occurs at $\tau_{c} \simeq 70$.

As one is dealing with fluctuations their amplitude is small in general, so their time decay can be calculated from linear hydrodynamic modes Ref. [⿰亻弋 , provided the fluctuations are stable. However, in the undriven IHS fluid the density fluctuations are growing exponentially like $\exp (\epsilon \tau)$ (clustering instability) [2]. The redeeming feature in the resolution of this paradox is that the energy only depends on products of modes, in which exponentially growing density fluctuations are balanced by exponentially decreasing temperature fluctuations.

In the case of inelastic hard spheres two colliding particles loose a fraction, $\epsilon \equiv 1-\alpha^{2}$, of their relative kinetic energy, where $\epsilon$ is the degree of inelasticity and $\alpha$ the coefficient of normal restitution. Particles move more and more parallel after every collision, and their random motion (temperature) decreases; i.e. the spatial fluctuations at shorter wavelength (which are rapidly damped out through viscosity and heat conduction) are not replenished with energy from the more microscopic degrees of freedom, as in the elastic case, where fluctuations at all wavelengths are kept at thermal noise level, fed by the randomizing elastic collisions. So, the dynamics selectively suppresses the shorter wave length components of the flow field, as is clearly illustrated in fig. 1a. After a time on the order of the mean free time $t_{0}$ between collisions, the surviving fraction of the energy $E(t)=(1 / N)\left\langle\sum_{i} \frac{1}{2} m v_{i}^{2}\right\rangle$ is totally stored in hydrodynamic modes (with wavenumbers $k<1$, where $k$ is the measured in units of inverse mean free path $l_{0}$ ) of the kinetic energy of the flow and the internal energy (granular temperature),

$$
E(t) \simeq \frac{1}{N} \int \mathrm{d} \mathbf{r}\left\{\frac{1}{2}\left\langle\rho(\mathbf{r}, t) u^{2}(\mathbf{r}, t)\right\rangle+\frac{d}{2}\langle n(\mathbf{r}, t) T(\mathbf{r}, t)\rangle\right\} .
$$

The subsequent decay of $E(t)$ is controlled by two different k-regimes [5]: (i) the elastic regime $(\epsilon<k<1)$ at short times (homogeneous cooling regime) with $t_{0}<t<t_{0} / \epsilon$, and (ii) 
the dissipative regime $(k<\epsilon)$ at large times $\left(t \gg t_{0} / \epsilon\right)$.

In the first regime the decay is controlled by the HCS, where $n(\mathbf{r}, t)=n, \mathbf{u}(\mathbf{r}, t)=0$ and a homogeneous temperature, decaying according to Haff's cooling law,

$$
T(t)=\frac{T_{0}}{\left(1+\gamma_{0} t / t_{0}\right)^{2}} \equiv T_{0} \exp \left(-2 \gamma_{0} \tau\right),
$$

where $E_{0}=\frac{d}{2} T_{0}$ is the initial (equilibrium) energy per particle, $\gamma_{0}=\epsilon / 2 d$, and $t_{0}=1 / \omega\left(T_{0}\right)$ is the mean free time with collision frequency $\omega\left(T_{0}\right)$ in the initial state. The second equality in eq. (2) defines the average number of collisions per particle within a time $t$. It is obtained by integrating $d \tau=\omega(T(t)) d t$, where $\omega(T) \sim \sqrt{T} / l_{0}$ with a temperature independent mean free path $l_{0}$, given by the Enskog theory for a dense system of hard spheres $(d=2,3)$. Haff's law is illustrated in figs. 2a,b by the straight lines, labeled 1 . The HCS is essentially an adiabatically changing equilibrium state, parametrized by $\{n, \mathbf{u}=0, T(t)\}$.

To evaluate the contribution of the long wavelength fluctuations, we linearize the fields in eq. (11) around the HCS, where $\mathbf{u}(\mathbf{r}, t), \delta n(\mathbf{r}, t)=n(\mathbf{r}, t)-n$ and $\delta T(\mathbf{r}, t)=T(\mathbf{r}, t)-T(t)$, are small (at least their combined effects; see redeeming features below), and consider Fourier modes. Retaining up to quadratic terms yields,

$$
E(t)=\frac{d}{2} T(t)+\frac{1}{2 N} \int \frac{\mathrm{d} \mathbf{k}}{\left(2 \pi l_{0}\right)^{d}}\left\{\rho\left\langle|\mathbf{u}(\mathbf{k}, \tau)|^{2}\right\rangle+d\langle\delta n(\mathbf{k}, \tau) \delta T(-\mathbf{k}, \tau\rangle\},\right.
$$

where $T(t)$ is given by Haff's law. The first term in the integrand is proportional to the structure factors $\left[(d-1) S_{\perp}(k, \tau)+S_{\|}(k, \tau)\right]$, and the second one to $S_{n T}(k, \tau)$, where the subscript $\|$ refers to the longitudinal component of $\mathbf{u}$ and the subscript $\perp$ to the transverse ones.

According to the theory of ref. [3a,b], the fluctuations around the HCS are described by fluctuating hydrodynamic Langevin equations, and obey an 'adiabatic' fluctuation dissipation theorem. The results from the structure factors, $S_{i j}(\mathbf{k}, \tau)$, obtained by numerically integrating the fluctuating hydrodynamic equations of ref. [3b], could be integrated over the full hydrodynamic range of wave numbers to obtain the decay of $E(t)$ for all times $t>t_{0}$.

Here, however, we present an analytic method that enables us to calculate the asymptotic decay for $t \gg t_{0} / \epsilon$ explicitly. For such times only modes in the dissipative regime with $k \ll \epsilon$ will contribute to the integrands in (3). In this regime, the dispersion relations for the modes, relative to the HCS, are well known [5,3c].

The structure factors for the $(d-1)$ transverse components $u_{\perp \alpha}$ and the longitudinal one $u_{l}$ in the range $k \ll \gamma_{0} \sim \epsilon$ can be deduced from ref. [3b], and yield,

$$
V^{-1}\left\langle\left|u_{\perp \alpha}(k, \tau)\right|^{2}\right\rangle=\left(T_{0} / \rho\right) e^{-2 \Delta_{\perp} k^{2} \tau}\left(1+\Delta_{\perp} k^{2} / \gamma_{0}\right),
$$

with a diffusivity $\Delta_{\perp}=\nu / l_{0}^{2} \omega$, where $\nu=\eta / \rho$ is the kinematic viscosity. The longitudinal structure factor is given by a similar expression with $\Delta_{\perp}$ replaced by $\Delta_{\|}$. The explicit form of the longitudinal diffusivity (given in ref. [3b] as $\Delta_{\|}=\gamma_{0} \xi_{\|}^{2} / l_{0}^{2}$ ) is not needed here. One can verify from the explicit expressions that $\Delta_{\|}>\Delta_{\perp}$, and even $\Delta_{\|} \gg \Delta_{\perp}$ for $\gamma_{0} \rightarrow 0$.

It should be noted that the dimensionless diffusivities, $\Delta_{\perp}$ and $\Delta_{\|}$, do not depend on the local temperature, since transport coefficients and collision frequencies are proportional to $\sqrt{T}$.

By inserting eq. (4) into (3) and performing the k-integrals, one easily finds that the contributions $E_{u u}(t)$ of the term $\left\langle|u|^{2}\right\rangle$ to eq. (3) decay as,

$$
E_{u u}(t) \simeq \frac{T_{0}}{2 n l_{0}^{d}}\left\{\frac{d-1}{\left(\Delta_{\perp}\right)^{d / 2}}+\frac{1}{\left(\Delta_{\|}\right)^{d / 2}}\right\}\left(\frac{1}{8 \pi \tau}\right)^{d / 2} .
$$


Next, we consider the contributions $E_{n T}(t)$ in (3) from the $n-T$ fluctuations. Calculation of the time dependence of $\delta n(\mathbf{k}, \tau)$ and $\delta T(\mathbf{k}, \tau)$ in the dissipative regime is in principle straightforward, but technically much more involved. One solves the initial value problem for the coupled linearized hydrodynamic equations by determining its eigenvalues (to $\mathcal{O}\left(k^{2}\right)$-terms included), and eigenvectors (to $\mathcal{O}(k)$-terms). The result for $k \ll \epsilon$ is,

$$
\begin{aligned}
& \delta n(\mathbf{k}, \tau) \simeq \frac{i k}{\gamma_{0}} C_{1} e^{\left(\gamma_{0}-\Delta_{\|} k^{2}\right) \tau} u_{l}(\mathbf{k}, 0)+\cdots \\
& \delta T(\mathbf{k}, \tau) \simeq \frac{i k}{\gamma_{0}} C_{2} e^{-\left(\gamma_{0}+\Delta_{\|} k^{2}\right) \tau} u_{l}(\mathbf{k}, 0)+\cdots,
\end{aligned}
$$

where the dots represent subdominant decaying terms, and $C_{n}(n=1,2)$ are some constants. The technical details are available through ref. [6].

The first equation in (6) exhibits the clustering instability. It shows that the density fluctuation is growing at an exponential rate. We are not aware of any publication on a freely evolving rapid granular flows or IHS fluids, that explicitly demonstrate how the clustering instability is driven through the coupling to the long wavelength longitudinal velocity fluctuations - except for the closely related nonlinear slaving mechanism between density and flow field, discussed by Goldhirsch et al. [2]-, whereas all other fluctuations remain bounded by their values in the initial thermal equilibrium state, i.e. the fluctuations in the flow field decay diffusively at rates $\Delta_{\perp} k^{2}$ and $\Delta_{\|} k^{2}$, and those in the temperature decay rapidly at a rate $\left(\gamma_{0}+\Delta_{\|} k^{2}\right)$.

The above discussion also implies that an incompressible granular flow $(\nabla \cdot \mathbf{u}=0)$ would not exhibit a clustering instability, at least not in a linear stability analysis. Moreover, an initial density fluctuation $\delta n(\mathbf{k}, 0)$ would not cause any clustering instability. Furthermore, we observe that $\langle\delta n(\mathbf{k}, \tau) \delta T(-\mathbf{k}, \tau)\rangle$ remains bounded, because the exponential increase of the first factor is balanced by an exponential decrease of the second. The resulting integral yields $E_{n T}(t) \propto \mathcal{O}\left(\tau^{-d / 2-1}\right)$, which is a subleading asymptotic term when compared to (5).

It can be shown similarly that the three mode contribution $E_{\text {nuu }}(t)$, neglected in the transition from eq. (11) to (3) remains bounded, and decays as $\tau^{-d}$, again a subleading correction. The dominant large time contribution $E_{u u}(t)$ is plotted in figs. 2a,b, where the lines labeled 2 and 3 represent the contributions of the transverse and longitudinal modes, respectively. The former contribution is a factor $e^{3} \simeq 20$ larger than the latter.

The result (6) also shows that there is no balancing of unstable density fluctuations in the structure factor $S_{n n}(k, \tau) \sim\left\langle|\delta n(\mathbf{k}, \tau)|^{2}\right\rangle$. Consequently, the predictions of the hydrodynamic Langevin equations for $S_{n n}$ breakdown at the crossover from the homogeneous cooling regime, as explained in ref. [3b], whereas those for $S_{\perp}, S_{\|}$and $S_{n T}$, used in this paper are expected to remain valid until far into the nonlinear clustering regime.

In summary, in the decay of the total energy $E(t)$, one can distinguish two different behaviors: homogeneous cooling for $\tau<\tau_{c}$, where $E_{\mathrm{HCS}}(t) \simeq \frac{d}{2} T_{0} \exp \left(-2 \gamma_{0} \tau\right)$ is given by Haff's law, and a diffusive decay for $\tau>\tau_{c}$, where $E(t) \simeq E_{u u}(\tau)$ is solely determined by the flow field, all long wavelength components of which decay diffusively. The equality $E_{u u}=E_{\mathrm{HCS}}$ defines the crossover time $\tau_{c}$, typical values of which are listed in the figure captions and table 1 .

Before comparing the preceding results with MD simulations, a final comment about the relation between the 'internal' kinetic time $\tau$ and the 'external' time $t$ is needed: in the homogeneous cooling state the relation between both times is correctly predicted by $\tau=$ $\left(1 / \gamma_{0}\right) \ln \left(1+\gamma_{0} t / t_{0}\right)$ in eq. (2). In the nonlinear clustering regime the relation between both times is not understood. The value of $\tau(t)$, as measured in the computer simulations for $\tau>\tau_{c}$, increases faster than those given by (2). For the cases corresponding to figs. 1a,b the time $\tau$ 
becomes roughly linear in $t$ for $\tau$ larger than 80 .

In fig. 2a the theoretical prediction for $E(t)$ is compared with computer simulations in a dense system $(\phi=0.245)$ of $N=50000$ IHS particles at low dissipation $(\alpha=0.9)$. The density or coverage is defined as $\phi=\frac{1}{4} \pi N \sigma^{2} / L^{2}$, where $\sigma$ is the diameter of a disk. The agreement remains good until far into the nonlinear clustering regime with $\tau \gg \tau_{c} \simeq 70$ (see fig. 1 la,b). The density field $n(\mathbf{r}, t)$ contains small $(\tau=80)$ and large $(\tau=160$; see fig. 1 $\mathrm{b}$ ) spatial inhomogeneities. The flow field shows both at $\tau=80$ (see fig. 1 1a) and at $\tau=160$ well-developed vortex patterns. To describe the crossover regime around $\tau_{c}$ one would have to evaluate $E(t)$ in the total hydrodynamic time regime $\left(t>t_{0}\right)$, by performing the numerical calculations described in the paragraph below eq. (3). The same good agreement is found at small inelasticities $(\alpha=0.975,0.9)$ for all densities $(\phi=0.05,0.11,0.245,0.4)$ in the larger systems with $N=50000$ particles, as summarized in Table 1 . When the number of particles is reduced to $N=20000$ and $N=5000$, the deviations steadily increase, suggesting finite size effects.
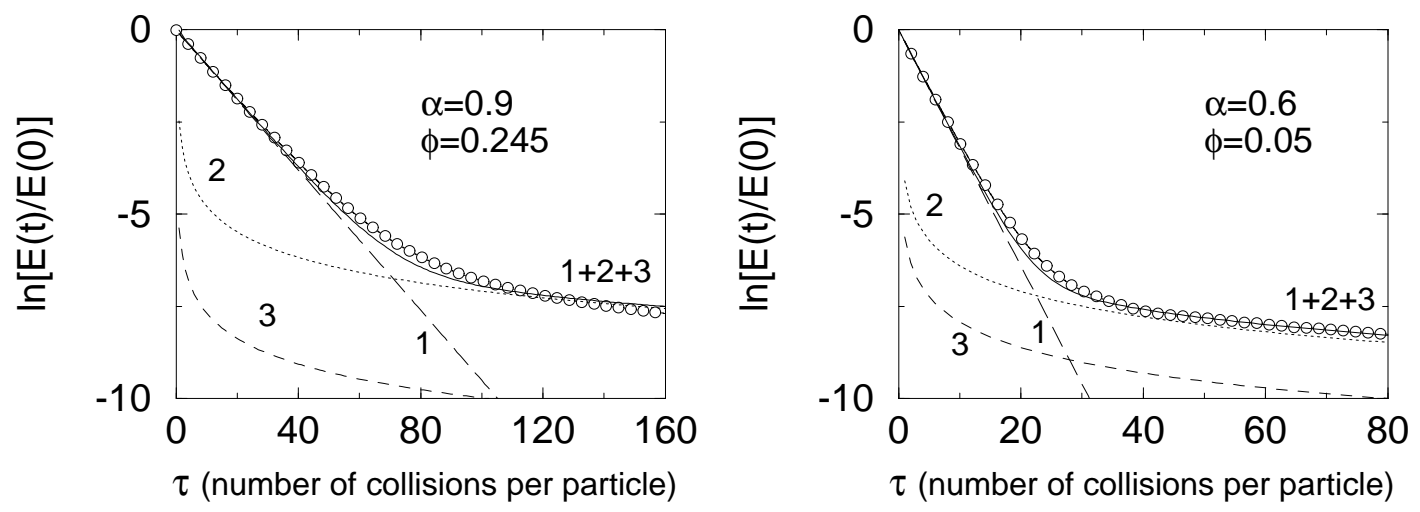

Fig. 2. -

Energy decay $E(t)$ vs. $\tau$ in simulations (o), with $N=50000$ IHS, (a) at low inelasticity $\left(\alpha=0.9, \phi=0.245, \tau_{c} \simeq 70\right)$, and (b) at high inelasticity $\left(\alpha=0.6, \phi=0.05, \tau_{c} \simeq 23\right)$, compared with theoretical prediction (labeled as $1+2+3$, solid line), containing homogeneous cooling (1), vorticity diffusion (2) and longitudinal diffusion (3). At the latest simulation times $\left(\tau_{l}\right.$ in Table 1$)$ both systems are in the nonlinear clustering regime (see fig. [ $1 \mathrm{~b}$ ).

Figure 2 b shows a similar plot of $E(t)$ in a low density system $(\phi=0.05)$ with a rather large inelasticity $(\alpha=0.6)$, and consequently a short crossover time $\tau_{c}=23$. There is very good agreement between theory and simulations over the whole time interval, until the time of the latest measurement $\tau_{l}=80$. At still larger inelasticities and higher densities $(\alpha=0.4, \phi=0.4)$ the theoretical prediction is an order of magnitude smaller than the simulated $E(t)$.

Table 1 also shows that the deviations at $\alpha=0.6$ between theory and simulations increase with increasing density (see $\phi=0.05,0.11,0.4$ ), suggesting that the increase of $\Delta(m, \alpha) / \Delta(m, 1)$ with $m=(\|, \perp)$ as $\alpha \rightarrow 0$ is much larger at high than at low density. A possible explanation might be that the Enskog theory for a dense IHS fluid, which is also based on molecular chaos, breaks down at higher inelasticities. The effects of dynamic correlations are expected to increase strongly with decreasing $\alpha$, when frequent inelastic collisions force 
EUROPHYSICS LETTERS

\begin{tabular}{|l|l|r|r|r|c|}
\hline \multicolumn{1}{|c|}{$\alpha$} & \multicolumn{1}{|c|}{$\phi$} & \multicolumn{1}{c|}{$\tau_{c}$} & \multicolumn{1}{c|}{$\tau_{\|}$} & \multicolumn{1}{c|}{$\tau_{l}$} & $E_{\text {sim }} / E_{\text {theor }}$ \\
\hline 0.975 & 0.245 & 340 & 27 & 500 & $0.65^{*}$ \\
\hline 0.9 & 0.245 & 70 & 240 & 160 & 0.8 \\
\hline 0.9 & 0.4 & 68 & 56 & 160 & 1 \\
\hline 0.6 & 0.05 & 23 & $10^{4}$ & 80 & 1 \\
\hline 0.6 & 0.11 & 19 & 3000 & 160 & 1.4 \\
\hline 0.6 & 0.4 & 15 & 150 & 80 & 4 \\
\hline 0.4 & 0.4 & 11 & 190 & 80 & 10 \\
\hline
\end{tabular}

TABLE I. - Comparison $E_{\text {sim }}\left(\tau_{l}\right)$ with $E_{\text {theor }}\left(\tau_{l}\right)\left({ }^{*} N=2 \times 10^{4}\right.$; in all others cases $\left.N=5 \times 10^{4}\right)$.

the particles to move more and more parallel. But so far ring kinetic theory 7 for calculating transport coefficients in IHS fluids has not yet been developed.

The present theory is only applicable to thermodynamically large systems, because in deriving (3) the k-sums have been replaced by $\mathbf{k}$-integrals. In the limit of small inelasticities $\left(\gamma_{0} \rightarrow 0\right)$, the smallest wave number is $k \sim 1 / L$, and the restriction $k \ll \gamma_{0}$ might be violated at small $L$.

For finite systems deviations from these predictions may occur because of interference effects through the periodic boundaries. In elastic hard sphere fluids such effects can be expected for times larger than the acoustic traversal time of the system. Here the fastest spreading mode is the longitudinal diffusion, and one may expect interference effects when the relevant diffusion length, $\xi_{\|} \equiv \sqrt{8 \Delta_{\|} \tau}$, satisfies $\xi_{\|} \gtrsim \frac{1}{2} L$. This implies $\tau \gtrsim \tau_{\|} \equiv L^{2} / 32 \Delta_{\|}$. For the simulations in fig. 1 and 2 a the time of the latest measurements, $\tau_{l}=160$, is of the order of $\tau_{\|}=240$, and the ratio $R\left(\tau_{l}\right) \equiv E_{\text {sim }} / E_{\text {theor }} \simeq 0.8$. In fig. $2 \mathrm{~b}, \tau_{l}=80 \ll \tau_{\|}=10^{4}$, and $\tau_{\|}=23$, and the ratio $R\left(\tau_{l}\right)=1$.

In summary, the mode coupling theory, applied to a freely evolving IHS fluid at small inelasticity $\epsilon$, explains how the hydrodynamic modes in the elastic range $(\epsilon \ll k<1)$ are responsible for Haff's homogeneous cooling law (2) at times $t \lesssim t_{0} / \epsilon$, and those in the dissipative range $(k \ll \epsilon)$ for the diffusive decay, $E(t) \sim \tau^{-d / 2}$ valid for $t \ll t_{0} / \epsilon$. In fact, the full range of hydrodynamic times $\left(t>t_{0}\right)$ can be evaluated numerically by applying the theory of refs. [3a,b] over the full range of hydrodynamic wavenumbers $(k<1)$. The solid line in figs. 2a,b, representing the sum of homogeneous cooling and transverse and longitudinal velocity diffusion, is simply an interpolation between the theoretical long and short time results. The agreement between theory and simulations is excellent over the large time interval from homogeneous cooling until far into the nonlinear clustering regime.

We also observe that the motion of the IHS fluid at the largest $\tau$-values (see fig. 1a,b and $2 \mathrm{a}, \mathrm{b})$ is almost 'frozen in', as the kinetic energy has typically decayed by a factor of order $10^{-3}$. Therefore, we do not expect a later crossover to truly nonlinear regime, which would imply that the decay law, derived here, is asymptotic indeed. At large inelasticities $(\alpha \lesssim 0.4)$ the present theory, which is based on a separation of time scales for small $\epsilon$, is not applicable, and the value of $E(t)$, observed in the simulations, is an order of magnitude larger than the prediction of the present theory.

Acknowledgements: It is a pleasure to thank D. Frenkel for an invaluable comment and helpful correspondence. We thank T.P.C. van Noije, J.A.G. Orza, I. Pagonabarraga and M. Hagen for stimulating discussions. The authors also acknowledge financial support from the Offices of International Relations of Universidad Complutense and Universiteit Utrecht. 
One of us (R.B.) acknowledges support to DGICYT (Spain) number PB94-0265.

\section{REFERENCES}

[1] P.K. Haff, J. Fluid Mech. 134, 401 (1983).

[2] I. Goldhirsch and G. Zanetti, Phys. Rev. Lett. 70, 1619 (1993); I. Goldhirsch, M-L Tan, and G. Zanetti, J.Sc. Computing 8, 1 (1993).

[3] (a) T.P.C. van Noije, M.H. Ernst, R. Brito and J.A.G. Orza, Phys. Rev. Lett. 79, 411 (1997).

(b) T.P.C. van Noije, M.H. Ernst and R. Brito, Phys. Rev. E 57, R4891 (1998).

(c) J.A.G. Orza, R. Brito, T.P.C. van Noije and M.H. Ernst, Int. J. Mod. Phys. C 8, 953 (1997).

[4] M.H. Ernst, E.H. Hauge and J.M.J. van Leeuwen, Phys. Rev. Lett. 25, 1245 (1970).

[5] S. McNamara, Phys. Fluids A 5, 3056 (1993).

[6] See web page: http://seneca.fis.ucm.es/brito/IHS.html.

[7] T.P.C. van Noije, M.H. Ernst and R. Brito, Physica A 251, 266 (1998). 